\title{
Neural correlates of reward-related response tendencies in an equiprobable Go/NoGo task
}

\author{
Onur Asci ${ }^{1} \cdot$ Senne Braem ${ }^{1,2} \cdot$ Haeme R. P. Park ${ }^{1}$ - C. Nico Boehler ${ }^{1}$ - Ruth M. Krebs ${ }^{1}$
}

Published online: 20 February 2019

(C) The Psychonomic Society, Inc. 2019

\begin{abstract}
Previous research has shown that motivational signals bias action over inaction, which may be due to putative inherent valenceaction mappings, similar to those observed in the emotional domain. In the present functional magnetic resonance imaging (fMRI) study we sought to investigate the neural underpinnings of such reward-related response tendencies, and in particular, how valence-action compatibility effects arising from predominant response tendencies are reflected at the neural level, and whether overlapping emotional valence amplifies these effects. To this end, we employed an equiprobable (50:50) Go/NoGo task in which reward (reward/no-reward) and response mode (Go/NoGo) were signaled by orthogonal features of number targets that were overlaid on emotional images (positive, neutral, negative). Reward-related targets led to response facilitation (faster Go responses) and impairment in withholding responses (more NoGo commission errors), consistent with a reward-induced action bias. This pattern was paralleled by modulations in the left dorsolateral prefrontal cortex (dlPFC), with increased activity in noreward as compared to reward-related Go trials, and the reversed pattern in NoGo trials. Albeit being processed in ventral visual areas, emotional background did not modulate performance in the present task, suggesting that irrelevant emotional information is globally outweighed by reward. In the current paradigm, which neither favors Go responses generally nor allows for differential preparation in Go versus NoGo trials, reward-related targets promote action over inaction. In turn, additional effort is needed to inhibit responses to these targets as well as to initiate responses to (less salient) no-reward targets, which may be considered as a downside of direct stimulus-reward associations.
\end{abstract}

Keywords Reward · Response bias · Go/NoGo · Emotional valence · fMRI

\section{Introduction}

The prospect of monetary incentives has been shown to affect cognitive processes and task performance in a variety of tasks. Typically, such reward manipulations lead to benefits in the form of simple response facilitation (e.g., Brian Knutson, Westdorp, Kaiser, \& Hommer, 2000), attentional discrimination (e.g., Engelmann, Damaraju, Padmala, \& Pessoa, 2009),

Electronic supplementary material The online version of this article (https://doi.org/10.3758/s13415-019-00692-5) contains supplementary material, which is available to authorized users.

Ruth M. Krebs

ruthmkrebs@gmail.com

1 Department of Experimental Psychology, Ghent University, Ghent, Belgium

2 Department of Experimental and Applied Psychology, Vrije Universiteit Brussel, Brussels, Belgium or improved cognitive control (e.g., Padmala \& Pessoa, 2011). However, there are also situations in which reward signals impair performance. This can be due to a mismatch between reward signals and current task goal, for example when reward-related stimuli are used as irrelevant distractors (e.g., Anderson, Laurent, \& Yantis, 2014; Hickey, Chelazzi, \& Theeuwes, 2010), or when one response mode is incentivized over another (e.g.,execution over inhibition; Padmala \& Pessoa, 2010). In the latter study, rewarding participants for fast and correct Go responses impaired their ability to withhold their response in Stop-signal trials. While such prioritization seems to work both ways in that one can also improve participants' ability to withhold a response by means of reward (Boehler, Hopf, Stoppel, \& Krebs, 2012; Boehler, Schevernels, Hopf, Stoppel, \& Krebs, 2014), there is also evidence of an inherent mapping between reward and response execution (or approach behavior), in that participants seem to learn this putative compatible valence-action mapping more easily than an incompatible one (e.g., Go-to-win vs. 
NoGo-to-win; Guitart-Masip et al., 2011; Guitart-Masip et al., 2012). Moreover, in the context of Pavlovian-Instrumental Transfer (PIT), it has been shown that stimuli that were previously associated with reward can bias response execution even if this goes against the current task goal, that is, in a NoGo context (Freeman, Razhas, \& Aron, 2014). Such response biases to reward-related stimuli are thought to be related to those observed in the emotional domain, where positive events facilitate approach, whereas negative events facilitate avoidance responses (Krieglmeyer, Deutsch, De Houwer, \& De Raedt, 2010; Phaf, Mohr, Rotteveel, \& Wicherts, 2014).

In the current study, we aimed to investigate the neural underpinnings of the putative response bias to reward-related stimuli by combining a rewarded Go/NoGo paradigm with fMRI. Specifically, we sought to (1) probe valence-action compatibility effects at the behavioral level in a (non-cued) Go/NoGo task, (2) test how these compatibility effects are represented at the neural level, and (3) whether overlapping and non-overlapping emotional valence amplifies these effects.

To approach these questions, we employed an equiprobable (50:50) Go/NoGo task in which reward prospect (Reward, NoReward) and response mode (Go, NoGo) were signaled trial-by-trial by orthogonal features of the target stimulus, and where performance in both Go and NoGo trials was equally important to obtain the reward. This stands in contrast to previous studies that have featured fixed valence-action mappings, entailed prioritization of one response mode over another, and/or in which reward contingencies were learned or conditioned (Freeman et al., 2014; Guitart-Masip et al., 2011; Padmala \& Pessoa, 2010). Importantly, an interaction between reward prospect and response mode (in the form of Go response facilitation and impaired NoGo performance in reward trials) would support the notion of a fairly automatic rewardtriggered response bias (hypothesis 1), even when valence and action features are randomly combined in each trial, and when it is not beneficial to favor Go over NoGo responses with regard to the expected outcome.

In turn, such a behavioral bias may be associated with valence-action compatibility effects at the cortical level, in that responding to compatible targets (Reward-Go and NoRewardNoGo) could be processed fairly effortlessly (consistent with the notion of an inherent bias), while incompatible targets (Reward-NoGo and NoReward-Go) may induce differential modulations in typical cognitive control regions in order to overcome prepotent response tendencies (hypothesis 2). Again, this would be in contrast to traditional reward cuing effects that tend to increase attention and cognitive control globally, solely resulting in performance improvements (Botvinick \& Braver, 2015; Braver et al., 2014; Krebs \& Woldorff, 2017).

Finally, considering overlapping valence-action mappings between the motivational and emotional domain, we tested whether emotional context (irrelevant background images) would further modulate these processes, in that positive and negative emotional context might enhance and reduce the reward-triggered response bias, respectively (hypothesis 3 ). Neurally, this might be reflected in activity modulations in valence-sensitive regions, such as the ventral striatum (VS) and the dopaminergic midbrain, in the form of enhanced activity when positive valence features are predominant, that is, concurrent reward and positive context, and reduced activity for the combination of no-reward and negative context. There is indeed evidence for such valence compatibility effects in that typical activity increases triggered by reward-predictive cues in a range of regions is diminished in anticipation of negative emotional events (Choi, Padmala, Spechler, \& Pessoa, 2014), and that the sensitivity to a negative event (as indexed by the electrophysiological P3 component) is reduced after reward cues (Wei, Wang, \& Ji, 2016). In contrast, when presented in the form of compound targets (in the absence of pre-cues), reward-related activity was increased by negative versus neutral information ( $\mathrm{Hu}$, Padmala, \& Pessoa, 2013), which might index increased overall saliency and/or cognitive demands.

To briefly pre-empt the results, the data provide evidence for hypothesis 1 (behavioral valence-action bias) and hypothesis 2 (compatibility effects at the neural level), while the third hypothesis was not confirmed (influence of emotional context).

\section{Methods}

\section{Participants}

Data from 24 participants without a history of neurological or psychiatric disease are reported in the present study (mean age $=23.42$ years, age range $=18-31,16$ females, all right-handed). One additional pilot participant (male) was excluded due to subsequent changes in the fMRI scanning protocol. Our sample size of 24 is well in line with previous neuroimaging studies that feature similar experimental factor combinations, with sample sizes ranging from 14 to 26 (including Choi et al., 2014; Freeman et al., 2014; Guitart-Masip et al., 2011; Hu et al., 2013), and we thus assume to have sufficient power to detect potential influences related to our manipulations. Participants were recruited from the student population of Ghent University. Upon arrival, they were informed about the task and fMRI procedures and gave their written informed consent to participate in the study. At the end of the session, each participant received a basic reimbursement of 35 Euros and an additional bonus of up to 9 Euros based on performance in reward trials. All experimental procedures were in accordance with the Declaration of Helsinki (1964) and its later 
amendments, and were approved by the Ethics Committee of the Ghent University Hospital.

\section{Task design and behavioral analysis}

Participants performed two runs of an equiprobable (50:50) Go/NoGo task with additional manipulations of reward prospect and emotional context (Fig. 1A). In each trial, a colored number (blue, RGB [0 130 255] or pink, RGB [230 10 200]) was displayed together with an emotional background image in the center of the screen for $1,000 \mathrm{~ms}$. Stimulus onset asynchrony (SOA) ranged between 2,250 and 9,000 $\mathrm{ms}$ in steps of 2,250 ms following a pseudo-exponential distribution of stimulus onsets that is recommended for event-related fMRI designs (Hinrichs et al., 2000), resulting in an average SOA of 3,683 ms. Target numbers ranged from 1 to 9 (except 5), and participants were instructed to respond to one set of numbers with their right index finger (e.g., number $<5$; Go trials), and not to respond to the other set of numbers (e.g., number $>5$; NoGo trials). Number color signaled the possibility of winning a monetary bonus (e.g. pink; Reward) or not (e.g., blue; NoReward). Participants were instructed regarding the colorreward contingencies before the experiment, which is in contrast to previous studies using learning or conditioning procedures (Freeman et al., 2014; Guitart-Masip et al., 2011). Moreover, participants were informed that correct performance referred to successful response execution in Go trials and successful response omission in NoGo trials. Hence, reward prospect was equally often linked to Go and NoGo target numbers across the experiment. Participants were told that they could earn a bonus of 5 cents for each correct reward trial (Go or NoGo), which could add up to a maximum of 9 Euro across the two runs. After each run, the accumulated amount was displayed on the screen.

A total of 180 emotional pictures (60 positive, 60 neutral, 60 negative) selected from the IAPS database (Lang, Bradley, \& Cuthbert, 2008) served as irrelevant background images. Positive and negative pictures were of opposite valence, differed significantly from the neutral baseline pictures both in valence and arousal $(\mathrm{p}<.001)$, and had similar arousal levels ( $p>05)$. Mean $( \pm$ S.D.) valence ratings on the scale of 1 (negative) to 9 (positive) were: Positive $=7.15 \pm 0.53$, negative $=3.33 \pm 0.59$, neutral $=5.56 \pm 0.55$; mean ( \pm S.D.) arousal ratings on the scale of 1 (low arousal) to 9 (high arousal) were: positive $=5.33 \pm 1.26$, negative $=5.70 \pm 0.74$, neutral $=4.26$ \pm 0.86 . Moreover, images were matched regarding the content across the three emotional categories to contain an equal amount of humans and animals. To counteract problems with respect to greater diversity in negative pictures (Alves, Koch, \& Unkelbach, 2017), we restricted these to fear-inducing pictures (conveyed by either humans or animals) and excluded pictures that primarily induced disgust. Each emotional background image was shown twice throughout the experiment.

All experimental factors (reward prospect, response mode, and emotional context) were orthogonal to each other, resulting in equally distributed trial numbers across all cells a

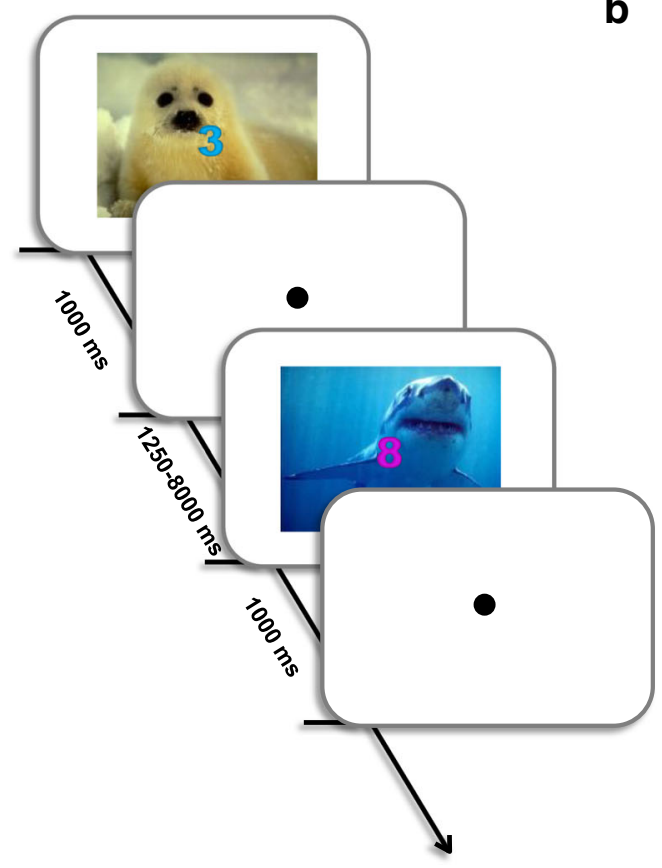

b

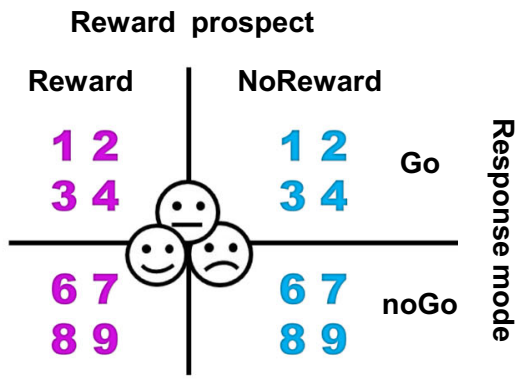

Fig. 1 Trial structure and task design. (A) Target numbers were overlaid on emotional background images. Number color signaled the reward prospect of the current trial (50:50), while number magnitude signaled the response mode (50:50). The emotional context (background image) was task-irrelevant. (B) The three experimental factors reward prospect (Reward, NoReward), response mode (Go, NoGo), and emotional context (positive, neutral, negative) were orthogonal to each other, resulting in a $2 \times 2 \times 3$ design 
of the design (Fig. 1B). Compound stimuli (colored number target plus background image) were displayed in the center of a white screen (visual angle $10 \times 6^{\circ}$ ), projected to a mirror mounted on the MR head coil. In the variable inter-trialinterval (1,250-8,000 ms), a black fixation dot was shown in the center of the white screen. Participants were instructed to keep their eyes in the center of the screen at all times (hence, either on the dot or on the target number) and to keep their head and body still throughout the entire experiment. Participants performed a total of 360 trials across two runs, yielding 30 trials per condition. Responses were recorded using an MR-compatible button box placed on the participants' right thigh, using the right index finger. Stimulus presentation and response acquisition was managed via Presentation software (http://neurobs.com/).

The experimental design is illustrated in Fig. 1B. In each trial, reward prospect (Reward, NoReward) and response mode (Go, NoGo) were combined in an orthogonal fashion, and both number-response mappings and color-reward mappings were counterbalanced across participants. Emotional context (positive, neutral, negative) of the background image was orthogonal to the other factors in the design and entirely irrelevant to the task. Picture sets used in reward and noreward trials were counterbalanced between participants. Response times (RTs) in Go trials (time-out 1,500 ms) were submitted to a $2 \times 3$ repeated-measures ANOVA (rANOVA) with factors reward prospect (Reward, NoReward) and emotional context (positive, neutral, negative). Error rates were submitted to a $2 \times 2 \times 3$ rANOVA with factors reward prospect (Reward, NoReward), response mode (Go, NoGo), and emotional context (positive, neutral, negative). Note that error rates reflect the percentage of response omissions in Go trials, and the percentage of commission errors in NoGo trials. Statistical analyses were performed in SPSS.

Before being positioned in the MR scanner, participants performed a short practice session of ten trials on a separate desktop computer to familiarize themselves with the Go/ NoGo task. Participants responded to Go targets by pressing the DOWN key on the keyboard with their right index finger. In contrast to the actual fMRI experiment, target color was irrelevant and thus not associated with any reward yet, and no emotional background images were shown. Instead, targets were overlaid on a gray placeholder square. Information regarding the color-reward contingencies and the emotional background pictures was given after the practice session.

\section{fMRI acquisition and preprocessing}

Data were acquired using a 3 Tesla Siemens Magnetom Trio MRI system (Siemens Medical Systems, Erlangen, Germany) with a standard 32-channel head coil. Before the functional scans, an anatomical T1-weighted 3D MPRAGE image (TR = $2,250 \mathrm{~ms}, \mathrm{TE}=4.18 \mathrm{~ms}, \mathrm{TI}=900 \mathrm{~ms}$, base resolution $=256$,
FoV $=256 \mathrm{~mm}$, flip angle $=9^{\circ}$, voxel size $=1 \times 1 \times 1 \mathrm{~mm}$ ) was acquired to enable spatial co-registration and normalization. Additionally, T2-weighted anatomical scans were acquired for 22 out of 24 participants $(\mathrm{TR}=11.49 \mathrm{~ms}$, TE $=$ $86 \mathrm{~ms}$, base resolution $=256, \mathrm{FoV}=220 \mathrm{~mm}$, flip angle $=$ $120^{\circ}$, voxel size $=1.2 \times 0.9 \times 1.2 \mathrm{~mm}$ ) at the end of the scan session. During the rewarded Go/NoGo task, two 11-min runs of T2*-weighted echo-planar images (EPIs) were acquired in 37 slices with an interleaved scanning order $(\mathrm{TR}=2,250 \mathrm{~ms}$, $\mathrm{TE}=30 \mathrm{~ms}$, base resolution $=64, \mathrm{FoV}=192 \mathrm{~mm}$, flip angle $=$ $80^{\circ}$, voxel size $=3 \times 3 \times 3 \mathrm{~mm}$, no inter-slice gap).

Images were preprocessed and further analyzed using the Statistical Parametric Mapping software (SPM8; University College, London, UK). Anatomical images (T1 and T2) were spatially normalized with reference to the SPM template images and resliced to a voxel size of $1 \times 1 \times 1 \mathrm{~mm}$. All functional EPIs were slice-time corrected, and realigned to the first acquired EPI after discarding the first four volumes to allow magnetization to reach equilibrium. Next, EPIs were normalized based on the T1-derived normalization parameters, resliced to a final voxel size of $3 \times 3 \times 3 \mathrm{~mm}$ (as acquired), and smoothed with an isotropic full-width half-maximum Gaussian kernel of $7 \mathrm{~mm}$. Head movements were generally small in the present sample, with the majority of participants moving less than $1 \mathrm{~mm}$ across the task, and four participants with a maximum of still less than $2 \mathrm{~mm}$.

During the experimental runs, skin conductance responses (SCRs) were recorded using an MR-compatible BIOPAC system (BIOPAC Systems, Inc., CA, USA). A summary of the procedures and SCR results can be found in the Supplemental Material (including Fig. S1).

\section{Voxel-wise fMRI analysis}

A standard two-stage procedure was used for the statistical analysis. For the first level analysis, blood oxygendependent (BOLD) responses were modeled by delta functions at stimulus onset, which were then convolved with a standard hemodynamic response function (HRF). The resulting general linear model (e.g., GLM, Friston et al., 1995) for each participant included 12 regressors representing correct trials of each experimental condition, one regressor for error trials, as well as six movement parameters derived from the realignment procedure. Before model estimation, a highpass filter of $128 \mathrm{~s}$ was applied to correct for slow drifts (Ashburner \& Friston, 1999). At the second level, activity maps from each participant and each condition were submitted to a voxel-wise random-effects analysis in the form of a flexible factorial model with factors reward prospect (Reward, NoReward), response mode (Go, NoGo), and emotional context (positive, neutral, negative). Activations were considered significant if they survived a family-wise error (FWE) correction at the cluster level with a threshold of $\mathrm{p}=.05$, based on a 
voxel-wise cluster-forming threshold of $p=.001$. Despite recent debates regarding FWE cluster-level correction (e.g., Eklund, Nichols, \& Knutsson, 2016), this procedure is considered to be sufficiently conservative for inferences at the cluster level (Flandin \& Friston, 2017). Coordinates of significant local maxima are reported in a standard stereotaxic reference space of the Montreal Neurological Institute (MNI) system. Anatomical labels are based on Automated Anatomical Labeling (AAL) implemented in SPM (for this step, SPM12 was used). To visualize interaction patterns observed in the voxel-wise analysis, parameter estimates (beta values) were extracted from all voxels within activated clusters and averaged for each condition using Marsbar (http:// marsbar.sourceforge.net/). No statistical tests were performed on these values to avoid circularity (Kriegeskorte, Simmons, Bellgowan, \& Baker, 2009).

\section{Anatomy-based region of interest (ROI) analysis}

To complement the voxel-wise analysis, we performed additional analyses in anatomically-defined regions of interest (ROIs) based on the a priori relevance of specific regions for our research questions. To this end, anatomical masks were drawn for the left and right ventral striatum (VS; encompassing the nucleus accumbens), based on the average T1 image of all participants using MRIcron (http://people.cas. sc.edu/rorden/mricron; Rorden \& Brett, 2000). These masks were further restricted by the average EPI image to account for potential signal loss in this area due to its proximity to air cavities. Following a similar procedure, masks for left and right substantia nigra and ventral tegmental area complex (SN/VTA) in the midbrain were drawn based on the average T2 image of 22 participants. From each of these masks, parameter estimates (beta values) were extracted and averaged across voxels and hemispheres for each condition using Marsbar. These estimates were submitted to a $2 \times 2 \times$ 3 rANOVA (SPSS) for bilateral VS and bilateral SN/VTA separately, mirroring the behavioral analysis.

\section{Results}

\section{Task performance}

Performance results are illustrated in Fig. 2. The $2 \times 3$ rANOVA of RTs (Go trials only) revealed a main effect of reward prospect $(\mathrm{F}(1,23)=59.84, \mathrm{p}<.001)$ with faster responses in Reward as compared to NoReward trials. The results of the $2 \times 2 \times 3$ rANOVA on error rates (including both Go and NoGo trials) yielded significant main effects of reward prospect $(\mathrm{F}(1,23)=17.91, \mathrm{p}<.001)$ and response mode $(\mathrm{F}(1,23)=22.89, \mathrm{p}<.001)$ as well as an interaction between these two factors $(\mathrm{F}(1,23)=22, \mathrm{p}<.001)$. Post hoc contrasts revealed that the interaction was driven by higher error rates for Reward compared to NoReward-NoGo trials $(\mathrm{t}(23)=4.68$, $\mathrm{p}<.001)$, and the reversed pattern for Go trials $(\mathrm{t}(23)=-2.81$, $\mathrm{p}=.010)$. In addition, error rates were significantly smaller in Reward-Go as compared to Reward-NoGo trials $(\mathrm{t}(23)=$ $5.01, \mathrm{p}<.001)$. The final contrast within this interaction (NoReward-Go - NoReward-NoGo) did not reach significance ( $p>$.7). No other main effects or interactions were found in RT and error-rate data (all p>.2), indicating that there was no systematic influence of emotion on task performance (see Supplemental Material for a related behavioral study).

Although participants were aware of reward and response contingencies from the start of the experiment and were not provided with trial-by-trial reward feedback, it is possible that the impact of reward and response mode manipulations grew stronger through the course of the experiment. We tested this by including "run" as an additional factor in the rANOVA of error rates. This analysis yielded no significant effects of runneither the main effect of run nor its interaction with the other factors (all $p>.2$ ) - indicating that the effects of interest are likely not the result of continuous learning throughout the experiment.

Considering the categorical nature of the error rate data and relatively low trial numbers, it may be argued that a rANOVA is not the optimal analysis approach (see, e.g., Jaeger, 2008). We hence conducted a follow-up analysis in the form of a generalized linear mixed-effects model approach using R. In addition to the main effects of all factors (coded as centered contrast), the model included the interaction between reward prospect and response mode, as well as subject as randomeffects factor. This analysis revealed a significant interaction between reward prospect and response mode $\left(\chi^{2}(1, N=24)=\right.$ $25.22, \mathrm{p}<.001)$, thereby confirming our initial interpretation of the accuracy data. We also observed a main effect of response mode $\left(\chi^{2}(1, N=24)=21.88, \mathrm{p}<.001\right)$. The main effects of reward prospect and emotional context were not significant (all $\mathrm{p}>.4$ ).

\section{Voxel-wise fMRI results}

Significant voxel-wise activations reflecting main effects of and interactions between our experimental factors are listed in Table 1 (FWE-corrected cluster level threshold $\mathrm{p}=.05$, auxiliary uncorrected voxel-wise threshold $\mathrm{p}=.001)$. The most robust effects were observed when contrasting Go and NoGo trials. The Go versus NoGo contrast yielded widespread activations across cortical and subcortical regions, including left motor cortex (contralateral to response hand), bilateral post-central gyrus, medial frontal gyrus, anterior insula, caudate nucleus, temporal, and occipital regions. Conversely, the NoGo versus Go contrast primarily yielded activity in the right motor cortex (ipsilateral to the response hand), left inferior frontal gyrus, and superior occipital gyrus. Note that the 
a
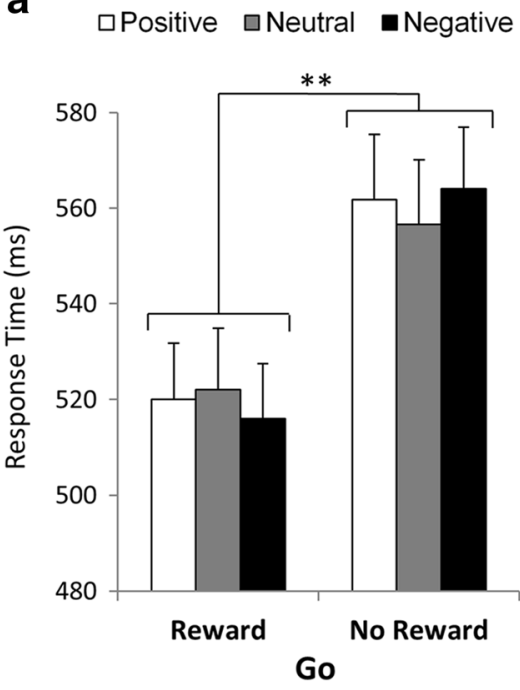

Fig. 2 Task performance results. (A) Go RTs were overall faster for reward-related targets compared to no-reward targets. (B) Error rates were significantly reduced in Reward-Go trials (virtually no response omissions overall and none for positive reward, hence the missing bar) b

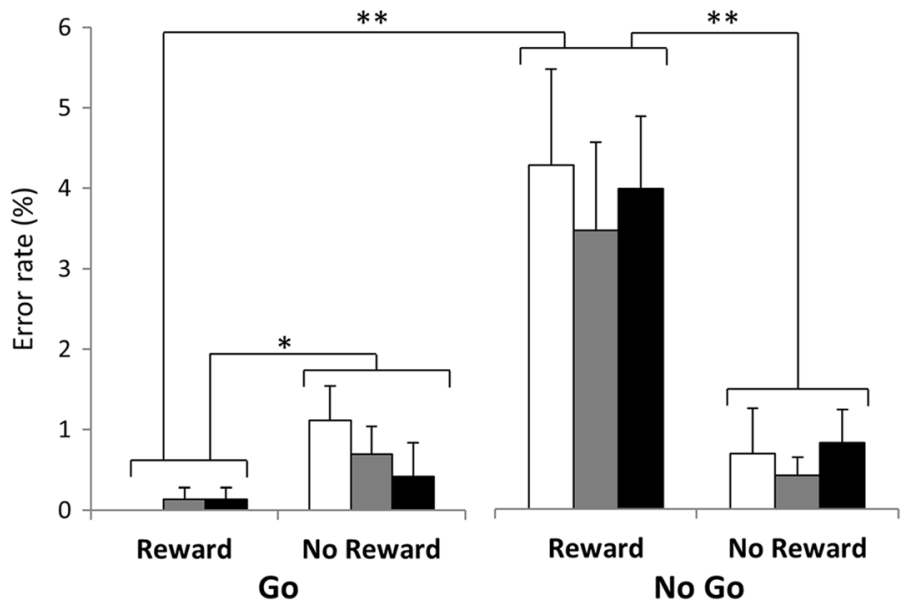

activations elicited by Go as compared to NoGo trials form a huge continuous cluster, which complicates the interpretation of individual subclusters regarding statistical significance. However, since our research question focuses on interactions between reward, emotion, and response mode, we draw no further inferences from the response mode main effects, other than that these activations are consistent with previous literature (see Discussion).

There was no global effect of reward prospect at the chosen threshold. Importantly, however, a cluster in the left dorsolateral prefrontal cortex (dlPFC, situated in BA46/9 and extending into BA10) featured an interaction pattern between reward prospect and response mode. Extracted parameter estimates from this cluster illustrate that the interaction was driven by increased activity in NoReward-Go as compared to Reward-Go trials (Fig. 3C), and the reversed pattern in NoGo trials, thus mirroring the interaction in the accuracy data. Note, however, that the voxelwise analysis is performed on correct trials only in order to avoid contributions from error processing. Hence, these modulations seem to be related to increased cognitive control to ensure correct task performance in the face of putative incompatible valence-action signals, i.e., initiating a response in NoReward-Go trials and withholding a response in Reward-NoGo trials.

Finally, while emotional context did not further modulate the relationship between reward prospect and response mode in the whole-brain analysis, both positive and negative emotional background stimuli (as compared to neutral ones) were associated with increased activity in visual processing regions, first and foremost in the middle occipital and fusiform gyri. This seems to suggest that emotional information (or at least and increased in Reward-NoGo trials (more commission errors) as compared to the respective other trial types. Significance level: $\mathrm{p}<.001 * *, \mathrm{p}<.01 *$. Error bars represent the standard error (SE) of the mean

general arousal conveyed by the images) was processed at some level, but did not affect task performance.

\section{Anatomy-based ROI results}

To test for potential interactions between reward prospect, response mode, and emotional context that might not have been detected in the voxel-wise analysis, we performed an anatomy-based ROI analysis in the ventral striatum and the SN/VTA complex in the midbrain, regions that are known to be involved in value processing (Duzel et al., 2009; B. Knutson \& Cooper, 2005). When conducting a $2 \times 2 \times 3$ rANOVA with the parameter estimates extracted from the VS (Fig. 4A), we found a trend for a main effect of reward prospect $(\mathrm{F}(1,23)=3.45, \mathrm{p}=.076)$, with higher activity for Reward compared to NoReward trials, and a main effect of response mode $(\mathrm{F}(1,23)=10.93, \mathrm{p}=.003)$ with higher activity in Go compared to NoGo trials. The same analysis in the $\mathrm{SN} /$ VTA (Fig. 4B) revealed a main effect of response mode, with higher activity in Go as compared to NoGo trials $(F(1,23)=42.09, p<.001)$. No other main effects or interactions were observed in these ROIs (all $\mathrm{p}>$.2).

\section{Discussion}

Reward signals can substantially influence our behavior - yet, this is not to say that this is always beneficial. One example of this is a putative response (or action) bias to reward-related stimuli that can lead to commission errors when the current trial requires suppressing the response. Previously, such tendencies have been observed when reward and response 
Table 1 Whole-brain voxel-wise main effects and interactions

\begin{tabular}{|c|c|c|c|c|c|c|c|}
\hline \multirow[t]{2}{*}{ Contrast/region } & \multirow[t]{2}{*}{$\mathrm{H}$} & \multirow[t]{2}{*}{$\mathrm{k}$} & \multicolumn{3}{|c|}{ MNI coordinates } & \multicolumn{2}{|c|}{ T-value peak (cluster p-value*) } \\
\hline & & & $\mathrm{x}$ & $\mathrm{y}$ & z & & \\
\hline \multicolumn{8}{|l|}{ Reward $>$ NoReward n.s. } \\
\hline \multicolumn{8}{|l|}{ NoReward > Reward n.s. } \\
\hline \multicolumn{8}{|l|}{ Go $>$ NoGo } \\
\hline $\begin{array}{l}\text { Pre-central/post-central gyrus, extending to Medial frontal } \\
\text { gyrus, Anterior insula, Caudate nucleus }\end{array}$ & $\mathrm{L}$ & 11361 & -57 & -22 & 46 & 16.69 & $(<.001)$ \\
\hline Cerebellum lobule 6 & $\mathrm{R}$ & & 18 & -55 & -20 & 16.42 & \\
\hline Rolandic operculum & $\mathrm{L}$ & & -51 & -22 & 19 & 16.13 & \\
\hline \multirow[t]{2}{*}{ Supramarginal gyrus } & $\mathrm{R}$ & 955 & 60 & -16 & 22 & 7.46 & $(<.001)$ \\
\hline & $\mathrm{R}$ & & 48 & -31 & 40 & 6.93 & \\
\hline Post-central gyrus & $\mathrm{R}$ & & 57 & -22 & 43 & 4.47 & \\
\hline \multirow[t]{2}{*}{ Calcarine fissure } & $\mathrm{R}$ & 91 & 30 & -67 & 7 & 4.21 & $(.016)$ \\
\hline & & & 21 & -70 & 7 & 4.09 & \\
\hline \multicolumn{8}{|l|}{ NoGo $>$ Go } \\
\hline \multirow[t]{3}{*}{ Pre-central gyrus } & $\mathrm{R}$ & 169 & 39 & -22 & 55 & 6.42 & $(.001)$ \\
\hline & $\mathrm{R}$ & & 51 & -16 & 58 & 5.38 & \\
\hline & $\mathrm{R}$ & & 39 & -25 & 67 & 4.93 & \\
\hline Superior occipital gyrus / Precuneus & $\mathrm{L}$ & 124 & -45 & -79 & 31 & 5.94 & $(.004)$ \\
\hline Inferior orbital frontal gyrus & $\mathrm{L}$ & 76 & -48 & 32 & -11 & 4.43 & $(.031)$ \\
\hline Inferior triangular frontal gyrus & $\mathrm{L}$ & & -54 & 35 & 4 & 4.11 & \\
\hline \multicolumn{8}{|l|}{$[$ Reward $<$ NoReward $] \times[$ Go $>$ NoGo $]$} \\
\hline \multirow[t]{3}{*}{ Middle frontal gyrus } & $\mathrm{L}$ & 82 & -36 & 32 & 25 & 4.03 & $(.024)$ \\
\hline & $\mathrm{L}$ & & -24 & 50 & 16 & 4.01 & \\
\hline & $\mathrm{L}$ & & -30 & 44 & 22 & 3.4 & \\
\hline \multicolumn{8}{|l|}{ Positive $>$ Neutral } \\
\hline Middle occipital gyrus & $\mathrm{R}$ & 227 & 39 & -79 & 22 & 5.21 & $(<.001)$ \\
\hline Fusiform gyrus & $\mathrm{L}$ & 122 & -30 & -52 & -8 & 5.18 & $(.004)$ \\
\hline Lingual gyrus & $\mathrm{L}$ & & -27 & -67 & -5 & 3.7 & \\
\hline Fusiform gyrus & $\mathrm{L}$ & & -30 & -34 & -17 & 3.43 & \\
\hline Middle occipital gyrus & $\mathrm{L}$ & 239 & -33 & -85 & 22 & 4.73 & $(<.001)$ \\
\hline \multirow[t]{2}{*}{ Superior occipital gyrus } & $\mathrm{L}$ & & -18 & -94 & 16 & 3.76 & \\
\hline & $\mathrm{L}$ & & -18 & -88 & 31 & 3.25 & \\
\hline Calcarine fissure & $\mathrm{R}$ & 198 & 18 & -88 & 10 & 4.59 & $(<.001)$ \\
\hline Fusiform gyrus & $\mathrm{R}$ & & 27 & -73 & -11 & 4.58 & \\
\hline Lingual gyrus & $\mathrm{R}$ & & 15 & -82 & -8 & 4.03 & \\
\hline \multicolumn{8}{|l|}{ Negative $>$ Neutral } \\
\hline Middle occipital gyrus & $\mathrm{L}$ & 147 & -48 & -70 & 1 & 4.85 & $(.002)$ \\
\hline Fusiform gyrus & $\mathrm{L}$ & & -39 & -49 & -11 & 3.78 & \\
\hline
\end{tabular}

$H$ hemisphere, $L$ left, $R$ right, $k$ cluster size

*Only clusters surviving an FWE (family-wise error)-corrected threshold of $\mathrm{p}=.05$ are shown (cluster-forming voxel-wise threshold $\mathrm{p}=.001$ )

requirements were signaled together by compound cues, or when reward was more strongly linked to responding than to not responding by design. In the present fMRI study we investigated the nature of such response biases to reward-related stimuli by employing a novel rewarded Go/NoGo paradigm in which reward prospect and response mode were signaled trialby-trial by orthogonal target features, and in which both Go and NoGo performance could be rewarded, thereby probing ad hoc processing of concurrent valence and action signals. This balanced design also allowed testing how potential valence-action compatibility effects arising from predominant response tendencies are processed at the neural level. In addition, we manipulated emotional context hypothesizing that overlapping (positive) and non-overlapping (negative) 
a

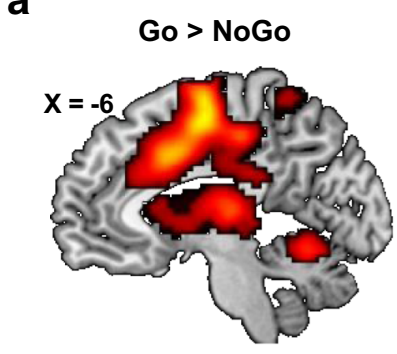

T-value $0 \longleftarrow 17$

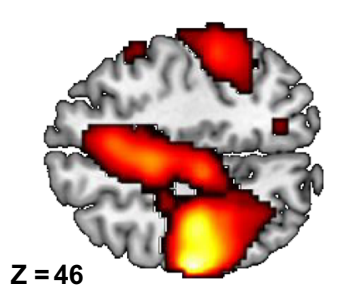

NoGo $>$ Go

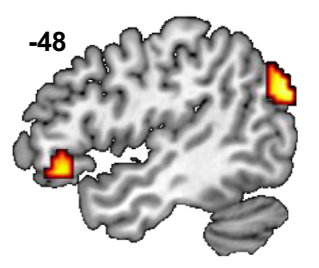

$0 \square 7$

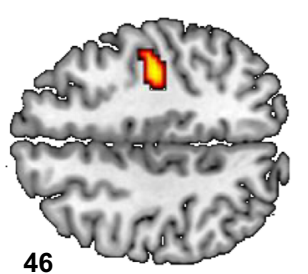

b
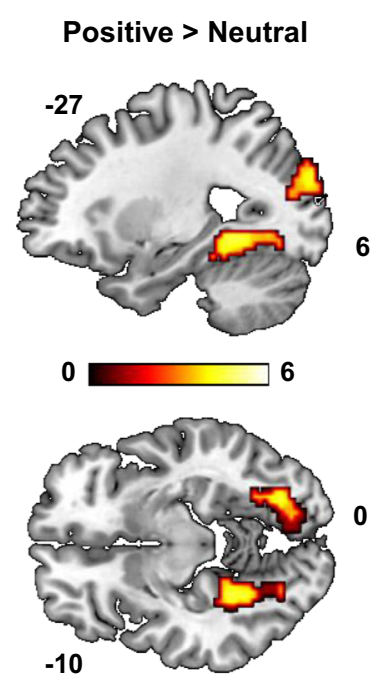

Negative $>$ Neutral

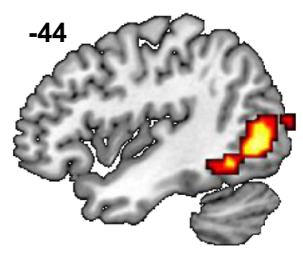

0

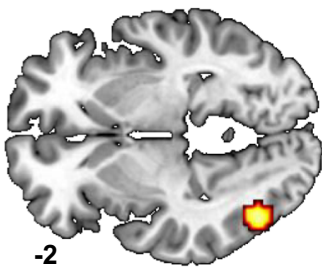

C

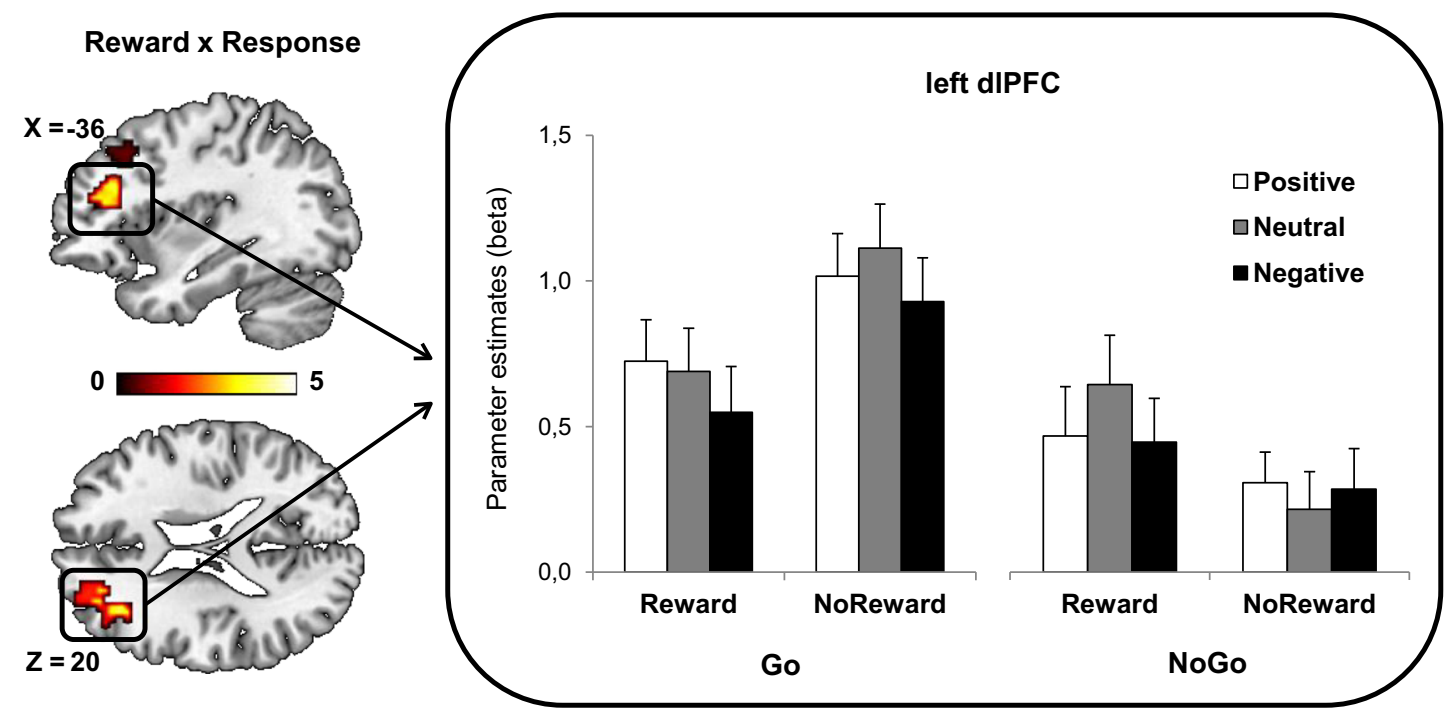

Fig. 3 Voxel-wise results. The factorial analysis revealed (A) main effects of response mode in a widespread network of cortical and subcortical regions, (B) main effects of emotional context in posterior visual areas, and $(\mathbf{C})$ an interaction between reward prospect and response mode in left

emotional valence signals could further augment and reduce reward-triggered response biases.

\section{Reward-triggered response bias}

We found that in comparison to no-reward targets, rewardrelated targets facilitated responses in Go trials (as indexed by shorter RTs and fewer response omissions), but impaired the suppression of responses in NoGo trials (as indexed by an increase in commission errors). This pattern strongly resembles the results of previous studies, which, however, employed fairly different designs (Freeman et al., 2014; Guitart-Masip et al., 2011; Guitart-Masip et al., 2012). Importantly, the majority of previous studies used advance cues to signal valence-
dIPFC. The interaction pattern is illustrated by plotting the parameter estimates averaged across all voxels in the left dlPFC cluster. Color bars represent T-value ranges. Error bars represent the standard error (SE) of the mean action mappings, which may already create differences during response preparation, in that preparing for a Go response is different to preparing for a NoGo response (Schevernels, Bombeke, Krebs, \& Boehler, 2016). Moreover, certain combinations of action and valence were mostly signaled by one unique stimulus, and were established via learning or conditioning procedures, which may have further strengthened their "inherent" association. The current design ameliorates these differences by combining reward- and response-mode features trial-by-trial in an orthogonal fashion and in the absence of advance cues or explicit learning (in contrast to, e.g., GuitartMasip et al., 2011). The current design also prevents the occurrence of differential global response strategies in that reward is equally linked to responding and not-responding 
a

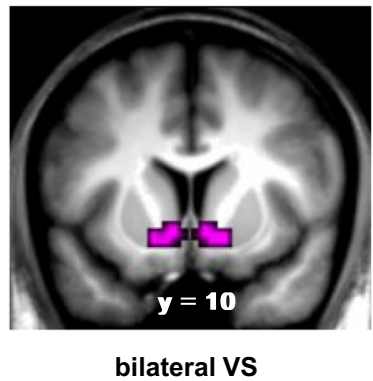

b

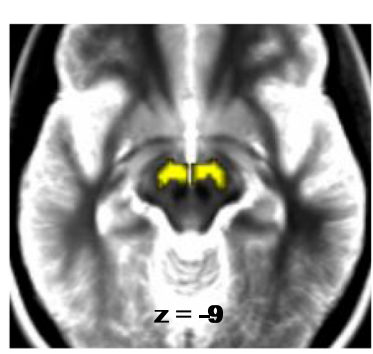

bilateral SN/VTA

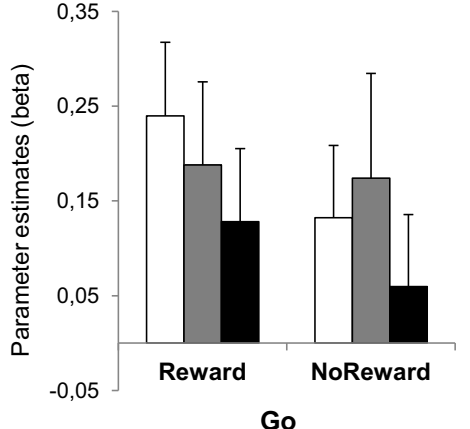

Go

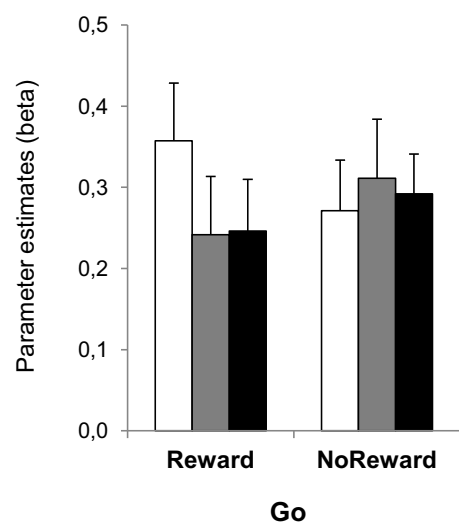

$\square$ Positive

aNeutral

- Negative

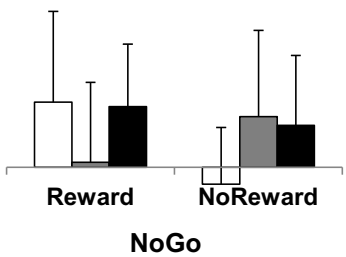

$\square$ Positive

$\square$ Neutral

- Negative

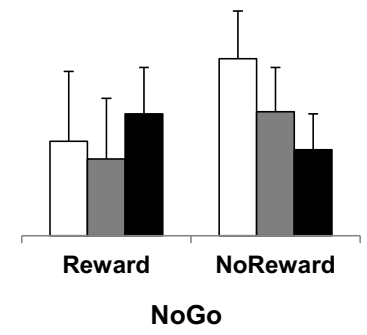

Fig. 4 Activity modulations within subcortical regions of interest (ROIs). (A) Activity in bilateral VS (pink mask) was mainly modulated by response mode - and by reward prospect at trend level. (B) Activity in bilateral SN/VTA (yellow mask) was modulated by response mode only.

(in contrast to, e.g., Freeman et al., 2014). Hence, the present study replicates previous observations of reward-triggered response biases in a novel task context that abolishes influences from strategic preparation and instead emphasizes automatic response capture. As such, the pattern resembles valenceaction biases that have been described in the emotional domain for decades (Krieglmeyer et al., 2010; Phaf et al., 2014; Zajonc, 1980). Interestingly, in contrast to the studies by Guitart-Masip et al. $(2011,2012)$, in which reward prospect was directly contrasted to loss prospect, we used no-reward trials as a baseline (cf. Freeman et al., 2014). The parallels in the results seem to suggest that incentive value is coded in a relative rather than in an absolute manner, and that no-reward trials may even be considered as negative events in the present task context.

\section{Controlling prepotent tendencies}

Based on the observed behavioral effects, we examined how compatibility effects of valence and action information are processed at the neural level. The first intriguing observation was that reward by itself did not lead to significant activity modulations anywhere in the cortex, suggesting that rewardrelated targets in the present paradigm did not trigger cognitive control processes or increased attention globally. This is
Despite numerical variations between conditions, emotional context did not affect activity in these regions significantly. Error bars represent the standard error (SE) of the mean

in contrast to more typical reward manipulations that signal reward prospect by advance cues (Krebs, Hopf, \& Boehler, 2016; Krebs \& Woldorff, 2017). Such cues do not require a response themselves, but serve to prepare differentially for the upcoming target by ramping up attention and cognitive control to ensure optimal task performance (Botvinick \& Braver, 2015; Braver et al., 2014; Pessoa \& Engelmann, 2010). The absence of such global activity increases in the present task may be surprising, but it certainly does not imply that participants did not process the reward information. First and foremost, their performance was substantially affected by reward. Moreover, reward prospect led to an activity increase at trendlevel in the ventral striatum, one of the most commonly reported regions across a diverse range of reward paradigms (for a review, see Sescousse, Caldu, Segura, \& Dreher, 2013). Therefore, the absence of elevated activity in attentional and cognitive control regions seems to suggest that behavioral modulations triggered by reward-related stimuli emerge in a fairly effortless manner (but see interaction pattern below).

As such, these influences could be described in terms of socalled response capture by salient inputs (Ridderinkhof, Forstmann, Wylie, \& van den Wildenberg, 2010), which is beneficial if the triggered response is in line with the task goal, but needs to be overcome if it is not. Intriguingly, the interaction between reward prospect and response mode that we 
observed in the left dlPFC is consistent with this notion. The dlPFC is globally implicated in cognitive control to integrate stimulus processing and response selection with our goals, and is of particular importance in goal-directed action selection when facing conflicting inputs or predominant response tendencies that collide with the current task goal (Boschin, Mars, \& Buckley, 2017; Kerns, 2006; Miller \& Cohen, 2001; Ridderinkhof, van den Wildenberg, Segalowitz, \& Carter, 2004). While activity in this region was globally lower for reward as compared to no-reward trials, indexing lower cognitive control demands, activity was increased for trial types featuring incompatible combinations of valence and action information (Reward-NoGo and NoRewardGo) as compared to compatible combinations (Reward-Go and NoRewardNoGo). In particular, in Reward-NoGo trials, cognitive control may be increased to select inaction in the face of a salient reward signal, while in NoReward-Go trials, control is needed to initiate a response to a relatively unremarkable target stimulus.

While previous studies have investigated similar interactions between incentive valence and action requirements (Freeman et al., 2014; Guitart-Masip et al., 2011; GuitartMasip et al., 2012), this is (to the best of our knowledge) the first observation of how putative (incentive) valence-action compatibility is processed at the cortical level. Specifically, in the fMRI studies discussed above (Guitart-Masip et al., 2011; Guitart-Masip et al., 2012), analyses regarding the interaction between valence and action requirements were focused on subcortical and ventromedial prefrontal areas by means of a partial-head volume. The present results hence go beyond the coding of action and value information that was the focus of previous studies. Specifically, they illustrate the consequences of valence-action incompatibility in terms of cognitive control demands (as indexed by prefrontal activity modulations). That said, our study also provides a replication of the initial study by Guitart-Masip et al. (2011) with respect to action and value coding in the VS - which is remarkable given that BOLD activity is locked to different events (i.e., cues in the studies by Guitart-Masip and colleagues vs. targets in the current study) and different valence contrasts (i.e., Win/Lose vs. Reward/NoReward). Specifically, in both studies, we see additive effects of action and valence in the VS, and an overall dominance of action in both VS and SN/VTA. Whether the main effect of action is a mere reflection of motor-related processes or also related to value processing remains an open question, and should be addressed in future studies. Although the design combines reward prospect and response mode in an orthogonal fashion, the factors might not be entirely independent from a psychological perspective (e.g., "acting" might feel more rewarding than "not acting" in such a lab context).

Considering the underlying neural mechanisms of the reward-triggered bias and overcoming the bias, the PIT study by Freeman et al. (2014) discussed above is highly relevant, as they administered transcranial magnetic stimulation (TMS) over the primary motor cortex contralateral to the response hand to test the influence of reward signals on corticospinal excitability. They found a rapid increase in corticospinal excitability in Go trials featuring reward-related stimuli, which ultimately led to faster responses in these trials. Conversely, in NoGo trials, reward-related stimuli lead to a differential suppression of motor activation, suggesting that prepotent reward-triggered response tendencies are counteracted. This observation is in line with our own results, not only in terms of behavioral outcome, but also in terms of the underlying mechanism in that the activity modulations in left dlPFC could be the reflection of cognitive control processes that contribute to successful suppression of a prepotent response.

When interpreting the present results, it is important to note that the current paradigm used equiprobable Go/NoGo trials. This is in contrast to unbalanced Go/NoGo tasks (Go more frequent than NoGo) that induce high levels of motor prepotency. Specifically, a recent electroencephalography (EEG) study provides evidence that only fast-paced Go/NoGo tasks with frequent Go trials induce motor prepotency, and are hence associated with high inhibitory control demands (Wessel, 2018). This is reflected in our data in that NoGo trials are not associated with prototypical activity modulations in the inhibitory control network (Aron, Robbins, \& Poldrack, 2004; de Zubicaray, Andrew, Zelaya, Williams, \& Dumanoir, 2000; Simmonds, Pekar, \& Mostofsky, 2008). Importantly, in contrast to studying inhibitory processes as such, we sought to use the Go/NoGo paradigm to investigate prepotent response tendencies triggered by trial-based reward signals. In this context, equal Go/NoGo trial proportions are in our view a prerequisite for preventing complex interactions between trialbased and sustained motor prepotency. Moreover, the present paradigm is also balanced with regard to reward-response outcome probabilities, thereby ameliorating strategic prioritization of one response mode over the other in the context of reward. Nevertheless, it is possible that the instruction to respond fast slightly biases participants towards Go responses in that the speed criterion is unique for Go trials (but see Gomez, Perea, \& Ratcliff, 2007).

Another important consideration is that while the behavioral evidence for reward-triggered response biases is based on accuracy measures (virtually no omission errors in Go trials vs. more commission errors in NoGo trials), the fMRI analyses are based on correct trials only to ensure that activity modulations are not confounded with error processing. This means that activity modulations in Go trials eventually lead to correct response execution, while activity modulations in NoGo trials eventually lead to successfully withholding the response. Considering the specific interaction pattern in the left dIPFC, we would thus argue that increased activity in this 
region is related to increased cognitive control demands to perform the required response (or withhold the response) in the face of prepotent tendencies, rather than a reflection of response execution or inhibition in particular. Note that while such general cognitive control signals have been reported in both left and right dIPFC (Koechlin, Ody, \& Kouneiher, 2003; Ridderinkhof et al., 2004), there are some indications that certain tasks or stimulus materials, such as semantic material (here, number targets) tend to preferentially trigger activity in the left hemisphere (e.g., Stephan et al., 2003).

\section{Influence of emotional context}

Finally, considering the overlap in valence-action compatibility effects in the motivational and emotional domain, we wanted to test whether emotional context would further modulate the observed relationships. It was very clear that emotion had no noticeable influence on behavior - neither on its own terms, nor in interaction with reward and/or response mode. This was confirmed in a related behavioral study in an independent group of participants (see Supplemental Material). However, this is not to say that emotional valence was not processed. In the fMRI experiment, positive and negative background images led to activity increases in posterior visual areas, including middle occipital areas and fusiform gyrus, which is consistent with existing neuroimaging studies on emotional stimulus processing (Phan, Wager, Taylor, \& Liberzon, 2002). However, in contrast to our predictions, there were no indications for valence compatibility effects between emotion and reward anywhere in the brain (at least not on a conservative FWE-corrected threshold). That said, we did observe interactions between reward and emotion in the SCR data, albeit only at trend level. Numerically, we observed higher SCR counts in trials containing positive or negative images as compared to neutral ones, and differentially increased SCR amplitudes for negative images. These slight modulations were restricted to no-reward trials, potentially indicating that salient reward-related targets attenuate the influence of emotional background images on a trial-to-trial basis. However, this did not impact on participants' behavior.

Considering all data modalities (performance, fMRI, and SCR), we conclude that although there is some indication that emotional context was processed, the two other factors arguably dominated participants' responses on every level. The most obvious reason for this asymmetry seems to be the fact that reward and response information was relevant for participants in order to perform the task as good as possible and to obtain rewards. While research from the attentional domain suggests that even irrelevant emotional signals are processed automatically (e.g., Vuilleumier, Armony, Driver, \& Dolan, 2001), the presence of potentially salient stimuli in the center of the screen might have counteracted the putative automatic influence of emotional information. Specifically, a relatively small area in the center of the visual field contained all the relevant information, potentially narrowing attentional focus in a sustained manner. Consistent with this, it has been argued that the processing of emotional information is subject to attentional control and not entirely automatic (e.g., Pessoa, McKenna, Gutierrez, \& Ungerleider, 2002). These considerations notwithstanding, we speculate that more salient valence manipulations, such as electrical shocks (see, e.g., Hu et al., 2013), would have been more powerful and harder to ignore.

\section{Conclusion}

In the current paradigm, which neither favors Go responses generally nor allows for differential preparation in Go versus NoGo trials, reward-related targets bias action over inaction. In turn, additional effort is needed to inhibit responses to these targets as well as to initiate responses to (less salient) noreward targets - which may be considered as a downside of direct stimulus-reward associations.

Acknowledgements This study was supported by a starting grant of the European Research Council (ERC) under the Horizon 2020 framework (grant No. 636116 awarded to RMK). We thank E. De Loof and T. Hitomi for their support during data analysis.

Publisher's note Springer Nature remains neutral with regard to jurisdictional claims in published maps and institutional affiliations.

\section{References}

Alves, H., Koch, A., \& Unkelbach, C. (2017). Why Good Is More Alike Than Bad: Processing Implications. [Review]. Trends Cogn Sci, 21(2), 69-79. https://doi.org/10.1016/j.tics.2016.12.006

Anderson, B. A., Laurent, P. A., \& Yantis, S. (2014). Value-driven attentional priority signals in human basal ganglia and visual cortex. Brain Research, 1587, 88-96. https://doi.org/10.1016/j.brainres. 2014.08.062

Aron, A. R., Robbins, T. W., \& Poldrack, R. A. (2004). Inhibition and the right inferior frontal cortex. Trends Cogn Sci, 8(4), 170-177. https:// doi.org/10.1016/j.tics.2004.02.010

Ashburner, J., \& Friston, K. J. (1999). Nonlinear spatial normalization using basis functions. Hum Brain Mapp, 7(4), 254-266.

Boehler, C. N., Hopf, J. M., Stoppel, C. M., \& Krebs, R. M. (2012). Motivating inhibition - reward prospect speeds up response cancellation. Cognition, 125(3), 498-503. https://doi.org/10.1016/j. cognition.2012.07.018

Boehler, C. N., Schevernels, H., Hopf, J. M., Stoppel, C. M., \& Krebs, R. M. (2014). Reward prospect rapidly speeds up response inhibition via reactive control. Cogn Affect Behav Neurosci. https://doi.org/10. 3758/s13415-014-0251-5

Boschin, E. A., Mars, R. B., \& Buckley, M. J. (2017). Transcranial magnetic stimulation to dorsolateral prefrontal cortex affects conflictinduced behavioural adaptation in a Wisconsin Card Sorting Test analogue. Neuropsychologia, 94, 36-43. https://doi.org/10.1016/j. neuropsychologia.2016.11.015

Botvinick, M., \& Braver, T. (2015). Motivation and Cognitive Control: From Behavior to Neural Mechanism. Annual Review of Psychology 
66, 66, 83-113. https://doi.org/10.1146/annurev-psych-010814015044

Braver, T. S., Krug, M. K., Chiew, K. S., Kool, W., Westbrook, J. A., Clement, N. J., ... Somerville, L. H. (2014). Mechanisms of motivation-cognition interaction: challenges and opportunities. Cogn Affect Behav Neurosci. https://doi.org/10.3758/s13415-0140300-0

Choi, J. M., Padmala, S., Spechler, P., \& Pessoa, L. (2014). Pervasive competition between threat and reward in the brain. [Research Support, N.I.H., Extramural]. Soc Cogn Affect Neurosci, 9(6), 737750. https://doi.org/10.1093/scan/nst053

de Zubicaray, G. I., Andrew, C., Zelaya, F. O., Williams, S. C. R., \& Dumanoir, C. (2000). Motor response suppression and the prepotent tendency to respond: a parametric fMRI study. Neuropsychologia, 38(9), 1280-1291. https://doi.org/10.1016/S0028-3932(00)00033-6

Duzel, E., Bunzeck, N., Guitart-Masip, M., Wittmann, B., Schott, B. H., \& Tobler, P. N. (2009). Functional imaging of the human dopaminergic midbrain. Trends Neurosci, 32(6), 321-328.

Eklund, A., Nichols, T. E., \& Knutsson, H. (2016). Cluster failure: Why fMRI inferences for spatial extent have inflated false-positive rates. [Research Support, N.I.H., Extramural Research Support, Non-U.S. Gov't]. Proc Natl Acad Sci U S A, 113(28), 7900-7905. https://doi. org/10.1073/pnas.1602413113

Engelmann, J. B., Damaraju, E., Padmala, S., \& Pessoa, L. (2009). Combined effects of attention and motivation on visual task performance: transient and sustained motivational effects. Front Hum Neurosci, 3, 4.

Flandin, G., \& Friston, K. J. (2017). Analysis of family-wise error rates in statistical parametric mapping using random field theory. Hum Brain Mapp. https://doi.org/10.1002/hbm.23839

Freeman, S. M., Razhas, I., \& Aron, A. R. (2014). Top-Down Response Suppression Mitigates Action Tendencies Triggered by a Motivating Stimulus. Current Biology, 24(2), 212-216. https://doi.org/10.1016/ j.cub.2013.12.019

Friston, K., Holmes, A. P., Worsley, K. J., Poline, J. B., Frith, C. D., \& Frackowiak, R. S. (1995). Statistical Parametric Maps in Functional Imaging: A General Linear Approach. Human Brain Mapping, 2(4), 189-210.

Gomez, P., Perea, M., \& Ratcliff, R. (2007). A model of the Go/No-Go task. Journal of Experimental Psychology-General, 136(3), 389413. https://doi.org/10.1037/0096-3445.136.3.389

Guitart-Masip, M., Chowdhury, R., Sharot, T., Dayan, P., Duzel, E., \& Dolan, R. J. (2012). Action controls dopaminergic enhancement of reward representations. Proceedings of the National Academy of Sciences of the United States of America, 109(19), 7511-7516. https://doi.org/10.1073/pnas.1202229109

Guitart-Masip, M., Fuentemilla, L., Bach, D. R., Huys, Q. J., Dayan, P., Dolan, R. J., \& Duzel, E. (2011). Action dominates valence in anticipatory representations in the human striatum and dopaminergic midbrain. [Comparative Study Research Support, Non-U.S. Gov't]. J Neurosci, 31(21), 7867-7875. https://doi.org/10.1523/ JNEUROSCI.6376-10.2011

Hickey, C., Chelazzi, L., \& Theeuwes, J. (2010). Reward Changes Salience in Human Vision via the Anterior Cingulate. J Neurosci, 30(33), 11096-11103. https://doi.org/10.1523/jneurosci.1026-10. 2010

Hinrichs, H., Scholz, M., Tempelmann, C., Woldorff, M. G., Dale, A. M., \& Heinze, H.-J. (2000). Deconvolution of Event-Related fMRI Responses in Fast-Rate Experimental Designs: Tracking Amplitude Variations. Journal of Cognitive Neuroscience, 12(supplement 2), 76-89. https://doi.org/10.1162/089892900564082

Hu, K., Padmala, S., \& Pessoa, L. (2013). Interactions between reward and threat during visual processing. [Research Support, N.I.H., Extramural]. Neuropsychologia, 51(9), 1763-1772. https://doi.org/ 10.1016/j.neuropsychologia.2013.05.025
Jaeger, T. F. (2008). Categorical Data Analysis: Away from ANOVAs (transformation or not) and towards Logit Mixed Models. $J$ Mem Lang, 59(4), 434-446. https://doi.org/10.1016/j.jml.2007.11.007

Kerns, J. G. (2006). Anterior cingulate and prefrontal cortex activity in an FMR1 study of trial-to-trial adjustments on the Simon task. Neuroimage, 33(1), 399-405. https://doi.org/10.1016/j.neuroimage. 2006.06.012

Knutson, B., \& Cooper, J. C. (2005). Functional magnetic resonance imaging of reward prediction. Curr Opin Neurol, 18(4), 411-417.

Knutson, B., Westdorp, A., Kaiser, E., \& Hommer, D. (2000). FMRI Visualization of Brain Activity during a Monetary Incentive Delay Task. NeuroImage, 12(1), 20-27.

Koechlin, E., Ody, C., \& Kouneiher, F. (2003). The architecture of cognitive control in the human prefrontal cortex. Science, 302(5648), 1181-1185. https://doi.org/10.1126/science. 1088545

Krebs, R. M., Hopf, J. M., \& Boehler, C. N. (2016). Within-trial effects of stimulus-reward associations. In T. S. Braver (Ed.), Motivation \& Cognitive Control. New York, NY: Psychology Press, Routledge.

Krebs, R. M., \& Woldorff, M. G. (2017). Cognitive control and reward. In T. Egner (Ed.), Handbook of Cognitive Control. Chichester, West Sussex, UK: John Wiley \& Sons.

Kriegeskorte, N., Simmons, W. K., Bellgowan, P. S., \& Baker, C. I. (2009). Circular analysis in systems neuroscience: the dangers of double dipping. Nat Neurosci, 12(5), 535-540.

Krieglmeyer, R., Deutsch, R., De Houwer, J., \& De Raedt, R. (2010). Being Moved: Valence Activates Approach-Avoidance Behavior Independently of Evaluation and Approach-Avoidance Intentions. Psychological Science, 21(4), 607-613. https://doi.org/10.1177/ 0956797610365131

Lang, P. J., Bradley, M. M., \& Cuthbert, B. N. (2008). International affective picture system (IAPS): affective ratings of pictures and instruction manual. Technical Report A-8. Gainesville, FL: University of Florida.

Miller, E. K., \& Cohen, J. D. (2001). An integrative theory of prefrontal cortex function. Annu Rev Neurosci, 24, 167-202.

Padmala, S., \& Pessoa, L. (2010). Interactions between cognition and motivation during response inhibition. Neuropsychologia, 48(2), 558-565.

Padmala, S., \& Pessoa, L. (2011). Reward reduces conflict by enhancing attentional control and biasing visual cortical processing. [Research Support, N.I.H., Extramural Research Support, Non-U.S. Gov't]. J Cogn Neurosci, 23(11), 3419-3432. https://doi.org/10.1162/jocn_a 00011

Pessoa, L., \& Engelmann, J. B. (2010). Embedding reward signals into perception and cognition. Frontiers Neuroscience, 4(17).

Pessoa, L., McKenna, M., Gutierrez, E., \& Ungerleider, L. G. (2002). Neural processing of emotional faces requires attention. Proceedings of the National Academy of Sciences of the United States of America, 99(17), 11458-11463. https://doi.org/10.1073/ pnas. 172403899

Phaf, R. H., Mohr, S. E., Rotteveel, M., \& Wicherts, J. M. (2014). Approach, avoidance, and affect: a meta-analysis of approachavoidance tendencies in manual reaction time tasks. Frontiers in Psychology, 5. Artn 378 https://doi.org/10.3389/Fpsyg.2014.00378

Phan, K. L., Wager, T., Taylor, S. F., \& Liberzon, I. (2002). Functional neuroanatomy of emotion: A meta-analysis of emotion activation studies in PET and fMRI. Neuroimage, 16(2), 331-348. https://doi. org/10.1006/nimg.2002.1087

Ridderinkhof, K. R., Forstmann, B. U., Wylie, S. A., \& van den Wildenberg, W. P. (2010). Neurocognitive mechanisms of action control: resisting the call of the Sirens. Wiley Interdisciplinary Reviews Cognitive Science, 2, 174-192.

Ridderinkhof, K. R., van den Wildenberg, W. P., Segalowitz, S. J., \& Carter, C. S. (2004). Neurocognitive mechanisms of cognitive control: the role of prefrontal cortex in action selection, response 
inhibition, performance monitoring, and reward-based learning. Brain Cogn, 56(2), 129-140.

Rorden, C., \& Brett, M. (2000). Stereotaxic display of brain lesions. Behav Neurol, 12(4), 191-200.

Schevernels, H., Bombeke, K., Krebs, R. M., \& Boehler, C. N. (2016). Preparing for (valenced) action: The role of differential effort in the orthogonalized go/no-go task. Psychophysiology, 53(2), 186-197. https://doi.org/10.1111/psyp.12558

Sescousse, G., Caldu, X., Segura, B., \& Dreher, J. C. (2013). Processing of primary and secondary rewards: A quantitative meta-analysis and review of human functional neuroimaging studies. Neuroscience and Biobehavioral Reviews, 37(4), 681-696. https://doi.org/10. 1016/j.neubiorev.2013.02.002

Simmonds, D. J., Pekar, J. J., \& Mostofsky, S. H. (2008). Meta-analysis of Go/No-go tasks, demonstrating that fMRI activation associated with response inhibition is task-dependent. Neuropsychologia, 46(1), 224-232. https://doi.org/10.1016/j.neuropsychologia.2007. 07.015
Stephan, K. E., Marshall, J. C., Friston, K. J., Rowe, J. B., Ritzl, A., Zilles, K., \& Fink, G. R. (2003). Lateralized cognitive processes and lateralized task control in the human brain. Science, 301(5631), 384-386. https://doi.org/10.1126/science.1086025

Vuilleumier, P., Armony, J. L., Driver, J., \& Dolan, R. J. (2001). Effects of attention and emotion on face processing in the human brain: An event-related fMRI study. Neuron, 30(3), 829-841. https://doi.org/ 10.1016/S0896-6273(01)00328-2

Wei, P., Wang, D., \& Ji, L. Y. (2016). Reward expectation regulates brain responses to task-relevant and task-irrelevant emotional words: ERP evidence. Social Cognitive and Affective Neuroscience, 11(2), 191203. https://doi.org/10.1093/scan/nsv097

Wessel, J. R. (2018). Prepotent motor activity and inhibitory control demands in different variants of the go/no-go paradigm. Psychophysiology, 55(3). UNSP e12871 https://doi.org/10.1111/ psyp. 12871

Zajonc, R. B. (1980). Feeling and Thinking - Preferences Need No Inferences. American Psychologist, 35(2), 151-175. https://doi.org/ 10.1037/0003-066x.35.2.151 